Revista de Negócios_ISSN 1980.4431_vol. 18, n.1, p.25_33, 2013_DOI:10.7867/1980-

431.2013v18n1p25_33

Edição Especial: Produção de Conhecimento Científico em Marketing

\title{
Perspectivas e limites da pesquisa quantitativa na produção de conhecimento em Marketing: A metáfora do cadeado
}

Prospects and limitations of quantitative research in the knowledge production in Marketing: The padlock metaphor

\author{
Salomão Alencar de Farias \\ Universidade Federal de Pernambuco \\ PROPAD - Programa de Pós-Graduação em Administração \\ e-mail: saf@ufpe.br
}

Recebido em 15 de fevereiro de 2013. Aprovado em 12 de março de 2013

Editor Responsável: Edson Roberto Scharf, Dr.

Autor convidado para edição especial

\section{Resumo}

Este ensaio aborda tópicos relacionados à pesquisa quantitativa e sua contribuição para o avanço do conhecimento em marketing no Brasil. Para tanto, propõe a metáfora do cadeado, discutindo de maneira leve aspectos relevantes da abordagem quantitativa de investigação na área do marketing, e revelando que, por vezes, a segurança dos números pode ocultar os limites deste tipo de pesquisa. No início dos anos 90 esta abordagem dominou as publicações e a formação de pesquisadores nos programas de pós-graduação em administração e ainda hoje é maioria nos artigos apresentados em congressos e revistas acadêmicas no país. A contribuição da pesquisa quantitativa na produção do conhecimento em marketing é clara considerando o levantamento feito por pesquisadores brasileiros; no entanto, os limites da abordagem não podem ser negligenciados. $O$ texto caracteriza-se como uma reflexão e não se propõe a ser prescritivo, mas apenas instigar o leitor a repensar os limites e as perspectivas da pesquisa quantitativa no marketing.

Palavras-chave: Pesquisa quantitativa; Marketing; Metáfora do cadeado.

\footnotetext{
Abstract

This essay discusses topics related to quantitative research and its contribution to the
} 
advancement of knowledge in marketing in Brazil. We propose "the padlock metaphor", discussing in a soft way, the relevant aspects of this approach in marketing research, revealing that sometimes the safety of numbers can hide the limits of this type of research. It is noticed that in the early 90's this approach dominated the publications and training of researchers in graduate programs in business and still today, it continues to do it so in most papers presented at conferences and academic journals in the country. The contribution to the production of knowledge in marketing with use of quantitative research is clear considering the surveys conducted by Brazilian researchers and here presented, however the limits of the approach cannot be overlooked. The text is characterized as a reflection and is not intended to be prescriptive, but only urging the reader to rethink the limits and prospects of quantitative research in marketing.

Keywords: Quantitative research; Marketing; Padlock metaphor.

\section{Introdução}

Iniciando este ensaio, volto a um tema que apresentei em painel de abertura de um encontro de marketing da ANPAD, onde junto com outros colegas discutíamos a pesquisa do comportamento do consumidor no Brasil. Ao ser convidado para participar desta mesa, me recordo que inicialmente pensei em apresentar algo tradicional, baseado em uma revisão teórica estruturada, me apossando do conhecimento de colegas pesquisadores na área, tanto do Brasil quanto do exterior. Até que um insight me direcionou para outro caminho. Resolvi fazer o uso da metáfora para abordar as perspectivas de pesquisa qualitativa e quantitativa, considerando que isso poderia trazer luz a um tema já conhecido, mas apresentado com uma abordagem de simples compreensão. Dessa forma, a base da minha fala foi a então denominada 'metáfora do cadeado'. Esta nomenclatura causou surpresa a alguns, risos em outros e, felizmente, interesse de outros. Para Richardson (1994, p. 519) a metáfora pode ser vista como a "espinha dorsal da escrita das ciências sociais", segurando pesos, criando equilíbrio, relacionando partes de uma forma coerente e funcional como um todo. Na verdade, o que desejei comunicar foi que a pesquisa quantitativa é para o pesquisador um "cadeado fechado", que traz segurança com seus mecanismos de fechadura (números), na medida em que pode apresentar indicadores de segurança: erros, confiabilidades, força das relações, etc., que, de algum modo, tornam mais amarrados e pontuais os resultados de uma análise de dados. Também os procedimentos de amostragem e coleta de dados, se seguidos de uma forma sistemática, trariam segurança mais fácil de ser comprovada do que na pesquisa qualitativa, que apresenta mais explicitamente a subjetividade do pesquisador, enfraquecendo os mecanismos de segurança do positivismo presente nos procedimentos quantitativos.

Assim, com esta metáfora em mente, é possível indicar que os limites da pesquisa estão até onde o 'cadeado' deixa o pesquisador seguro do que está fazendo. Ao mesmo tempo em que traz segurança, um cadeado fechado limita o pesquisador, que deve seguir pressupostos teóricos e de mensuração de forma rigorosa, sem os insights subjetivos da abordagem qualitativa. Assim, a pesquisa qualitativa seria um 'cadeado aberto', pois traria segurança ao pesquisador (com a sistematização de procedimentos), mas também ofereceria saídas para caminhos não tradicionais de coleta e interpretação de dados, sem a necessidade de chegar a conclusões pontuais ou definitivas. Acrescente a isso que com o 'cadeado aberto', o pesquisador pode ousar mais em seus achados, sem a garantia numérica dos achados presentes na pesquisa quantitativa.

Então quais os limites da pesquisa quantitativa? Quais suas perspectivas? De que modo tem contribuído para a produção do conhecimento em marketing? Há uma preferência da pesquisa quantitativa nos programas de pós-graduação (mestrado e doutorado) no Brasil, em detrimento da qualitativa? Esses questionamentos são o mote deste ensaio. Não se pretende aqui 
apresentar um texto prescritivo relacionado à pesquisa quantitativa. Isso se encontra em livros de pesquisa de marketing ou metodologia de pesquisa. $\mathrm{O}$ intuito é refletir sobre a pesquisa quantitativa com uma perspectiva leve, indicando que foi com base nesta abordagem que os pesquisadores formados nos anos 90 pelos programas de doutorado em administração no Brasil contribuíram para a disseminação e avanço do conhecimento em marketing neste país, bem como para a formação de uma nova geração de pesquisadores que hoje está lecionando nos principais programas nacionais.

As informações que serão apresentadas nos próximos tópicos reforçam o papel da pesquisa quantitativa na produção do conhecimento em marketing no Brasil. $\mathrm{O}$ rigor nas análises estatísticas, as amostras com grande número de respondentes, por vezes representativas do universo e outras por conveniência, o acesso a softwares estatísticos específicos para as ciências sociais, os bancos de dados virtuais (com destaque inicial ao Proquest $\left.{ }^{\circledR}\right)$ e o barateamento dos computadores pessoais, possibilitaram o uso de técnicas estatísticas mais avançadas junto a bancos de dados que nos anos 80 tinham que fazer uso de mainframes para tal.

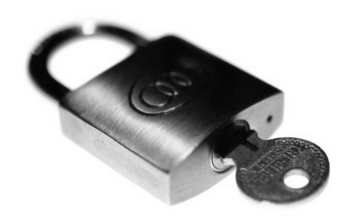

Pesquisa Quantitativa: cadeado fechado

- Rigor nas métricas, na coleta e análise de dados

- Parâmetros numéricos de segurança

- Rigidez em preceitos para aplicação de técnicas estatísticas

- Conclusões pontuais

- Objetividade por parte do pesquisador
A figura 1 ilustra dois cadeados com simbolismo de representação de segurança na abordagem da pesquisa quantitativa e qualitativa (o cadeado representa a segurança na pesquisa), considerando os pressupostos métodológicos de ambas as abordagens. A ilustração do cadeado é utilizada em diversas situações para representar segurança, como por exemplo, em sítios da internet onde o consumidor percebe risco com mais frequência, especialmente em transações financeiras. Aqui, apropria-se metaforicamente deste objeto com o intuito de transmitir que, como um cadeado, a pesquisa quantitativa com seus parâmetros e rigidez, traz segurança ao pesquisador e indica suas limitações com objetividade. De outro lado, na pesquisa qualitativa, ainda há segurança, mas o cadeado aparece aberto, pois há mais possibilidade de subjetividade na interpretação dos dados e nas conclusões. Ambos apresentam uma chave indicando que toda abordagem de pesquisa tem limites, e o leitor com conhecimento sobre os métodos pode indicar claramente os limites ou falhas de uma pesquisa, contestando os resultados apresentados.

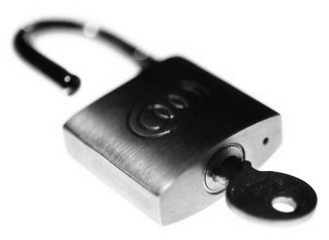

Pesquisa Qualitativa: cadeado aberto

- Rigor na sistematização

- Parâmetros de qualidade

- Flexibilidade nas análises

- Conclusões não definitivas

- Clara subjetividade do pesquisador

Figura 1 - A metáfora do cadeado 
O ensaio estrutura-se em quatro blocos principais onde tópicos como a qualidade da pesquisa, o papel da teoria, o processo de amostragem e a mensuração em pesquisa quantitativa são discutidos, para concluir com reflexões gerais sobre a temática central aqui colocada.

\section{A qualidade dos resultados publicados com base em estudos quantitativos}

Em 1978, Jabocy indicou que "uma grande proporção da literatura de pesquisa do consumidor (incluindo marketing) não vale o papel que é impressa ou o tempo que leva para ler" (p. 87). Passados 35 anos desta afirmação, será que isto ainda é válido? Considerando que boa parte da produção está disponível de forma digitalizada em sítios da web, talvez somente a perda de tempo se aplique, em termos comparativos sem, no entanto, discordar que alguns artigos apresentam ausência de qualidade por falta de cuidados básicos na condução da pesquisa e análise dos dados. Tem-se um cadeado defeituoso, que aparenta dar segurança ao pesquisador, mas uma avaliação cuidadosa indica falhas que comprometem os achados.

Um olhar na produção acadêmica brasileira revela uma evolução na qualidade da pesquisa em administração de modo geral, a partir da expansão dos cursos de doutorado e a formação diversificada do corpo docente destes programas, em instituições nacionais e internacionais. O treino científico, em especial nos métodos quantitativos de pesquisa nos anos 90, contribuiu para a consolidação desta abordagem em marketing na produção acadêmica nacional.

Em 1998 quando defendi minha tese de doutorado com o uso da modelagem de equações estruturais e posteriormente um artigo resultante desta recebeu o prêmio de melhor artigo no encontro ENANPAD na área de marketing, foi um indicativo da aceitação dos métodos quantitativos como mainstream. Àquela época, final dos anos 90, ainda não era comum o uso desta técnica nas dissertações e teses na área de marketing no Brasil. Com o acesso aos softwares mais amigáveis como o AMOS $®$, outros pesquisadores iniciaram seu uso, e hoje a modelagem de equações estruturais é bastante utilizada no teste de relações entre variáveis em pesquisas acadêmicas no Brasil. Por tempos, esta técnica não foi empregada nas ciências sociais devido a dificuldade de se especificar relações de causa e efeito, onde em outras ciências seria $o$ foco principal da mesma. As relações de causa e efeito nas ciências sociais por vezes são frágeis, pela própria natureza dos seus fenômenos. No entanto, aqui tem-se um cadeado sofisticado e poderoso na aparência, que pode ser aberto se os preceitos da técnica não forem atendidos em sua totalidade.

A modelagem de equações estruturais entusiasma por seu poder de análise, bem como pela "beleza" dos seus modelos complexos, considerando o grande número de relações avaliadas simultaneamente. A força aparente da análise, do modelo e de sua complexidade muitas vezes são responsáveis pela aceitação de um artigo para apresentação em congresso ou publicação em revista, somente pela aplicação da técnica em si (o 'cadeado' impressiona). Talvez um viés quantitativo do avaliador ou o desconhecimento deste dos requisitos levem a uma avaliação frouxa do trabalho. O destaque inicial a esta técnica multivariada deve-se ao fato do seu modismo e utilização intensa em pesquisas quantitativas na área de marketing no Brasil. Quando aplicada seguindo os cuidados e recomendações necessárias, relações múltiplas podem ser avaliadas em um único modelo, incrementando as possibilidades de teste da teoria, o 
que pode certamente, contribuir com avanços no conhecimento.

Recentemente, Mazzon e Hernandez (2013) apresentaram um levantamento da produção científica brasileira em marketing, onde no período de 2000 a 2009 preponderaram os estudos empíricos $(85,6 \%)$, incluindo anais de congressos e periódicos qualificados no mínimo com B2 no Qualis CAPES. Desse total, 58,3\% eram quantitativos (741 artigos no total) e $37,3 \%$ qualitativos. Isso revela que os estudos quantitativos ainda dominam a produção na área no Brasil. No entanto, o volume de trabalhos qualitativos tem avançado, indicando que os programas de pós-graduação no país estão também treinando seus pesquisadores em caminhos alternativos. Desse modo, especula-se que os estudos quantitativos contribuem mais com a produção do conhecimento na área de marketing, considerando o número de publicações em comparação aos qualitativos. Claro que aqui há um viés de simplificação de linearidade onde quantidade estaria associada à contribuição para o avanço do conhecimento, sem o mérito explicito da qualidade desta produção.

A dominância quantitativa ainda presente em grande parte dos programas de pós-graduação em administração no Brasil e certamente as exigências para a elaboração de uma tese e obtenção do título de doutor levam a resultados de pesquisa em sua maioria de qualidade, onde o recém-doutor constrói artigos com base no texto defendido, em coautoria com o orientador, que em muitos casos são contemplados com premiações nos encontros do ENANPAD e publicados em revistas com qualificação $\mathrm{B} 1, \mathrm{~A} 2$ e $\mathrm{A} 1$. Neste caso, espera-se que exista contribuição de qualidade para o conhecimento em marketing no país com base em estudos quantitativos.

\section{O Papel da Teoria}

Dando continuidade a metáfora do cadeado, a teoria faz parte do mecanismo de segurança do pesquisador, uma vez que o escopo desta dentro de uma determinada temática indica ao indivíduo os caminhos para a especificação de hipóteses e modelagem, quando necessário. A revisão teórica que apresenta o estado da arte do conhecimento na área que a pesquisa será realizada traz segurança e alicerça as decisões conduzidas no decorrer de um estudo científico. Sistematizar a teoria, definir o escopo dos construtos, conhecer em profundidade sobre $\mathrm{o}$ fenômeno investigado, esses passos trazem segurança inicial, a exemplo do uso de um cadeado que protege uma propriedade valiosa.

Vieira (1998, p. 9) analisou a publicação científica na área de marketing do ENANPAD e concluiu que o acadêmico de marketing brasileiro, "no âmbito do ENANPAD, usa mais livros do que periódicos como referências para a construção do quadro teórico-metodológico de suas análises e investigações científicas". Isso fragiliza o mecanismo de segurança teórica do "cadeado" do pesquisador. A produção em periódicos científicos é mais dinâmica e passa por avaliação de pares antes da publicação, assegurando de algum modo sua qualidade. Assim, o caminho, com exceções, deve ser o do uso de artigos científicos de periódicos de alta credibilidade, reforçando o mecanismo de segurança do cadeado da pesquisa quantitativa.

A base teórica é indispensável para a qualidade da pesquisa, seja esta qualitativa ou quantitativa. Modelos teóricos a serem testados por meio de análises multivariadas, em especial a modelagem de equações estruturais, devem necessariamente estar embasados em teoria. É importante atenção para não cair na tentação da teoria da moda ou na superficialidade de justificativas para relações teóricas ou, ainda, o uso de escalas previamente testadas para mensurar construtos sem, no entanto, considerar-se o 
aporte teórico que respalde as associações ou relações testadas. Na realidade, este ponto merece atenção especial, pois em se tratando da literatura acadêmica nacional, na maior parte das vezes o que ocorre é uma repetição (replicação) de estudos, com poucas alterações, como a inclusão de um construto ou a pesquisa em outro setor diferente do originalmente estudado. Mas será que isso limita ou invalida a contribuição da pesquisa quantitativa para o avanço do conhecimento em marketing no Brasil?

Em se tratando de experimentos, o uso da teoria para embasar os estudos, vale destacar o que Alba, Kraemer e Slongo (2012) comentaram: a revisão teórica apresentada em artigos nacionais publicados em periódicos é mais extensa do que as internacionais, mas não tem levado os autores nacionais a testar relações entre variáveis de forma contraintuitivas, "proposições que não parecem prováveis se analisarmos intuitivamente sob os pressupostos teóricos" (p. 10). Ou seja, a segurança trazida por esta etapa da pesquisa está limitando a criatividade dos pesquisadores.

\section{O Processo de Amostragem}

“-Estudantes são gente?". Escutei o questionamento de um professor quando cursei disciplina no doutorado, ao explicar o uso (ou abuso) de estudantes como amostra para quase todo tipo de pesquisa na área de marketing. Revisando textos publicados em diferentes revistas acadêmicas, incluindo aí aquelas de origem norte-americana, há uma tendência para o uso deste tipo de amostra. A facilidade de acesso aos respondentes, a disponibilidade de participar nas pesquisas (muitas vezes com bônus na avaliação em uma disciplina), os custos temporais e financeiros reduzidos levam os pesquisadores a adotarem em suas pesquisas os estudantes, em detrimento aos consumidores ditos "reais". Outra possibilidade é o uso da internet para acessar respondentes, que também apresentam limitações (e vantagens).

Alba, Kraemer e Slongo (2012) ao apresentarem um panorama da pesquisa experimental em marketing no Brasil, indicam que "a partir do ano 2000 as pesquisas que utilizam o método experimental na área de marketing começaram a se tornar mais frequentes" (p. 5). Acrescente a isso que o uso de simulações com consumidores reais ainda são mais "fáceis" no Brasil do que em países como os Estados Unidos, onde a questão da individualidade, do respeito à privacidade e do custo de usar amostras de consumidores - que não estudantes - é mais complicado do que no Brasil. Para exemplificar, Costa e Farias (2011) realizaram experimento com amostra de consumidores reais em um ambiente de varejo. Isso foi possível devido ao interesse de um dos dirigentes do negócio e na confiança deste em pesquisa acadêmica. No entanto, ressalta-se que nem sempre é fácil ter acesso a este tipo de amostra e o caminho mais fácil são os estudantes. Ate que ponto o uso de estudantes fragiliza o cadeado da pesquisa quantitativa? A resposta que ouvi em relação ao questionamento "os estudantes são gente?", foi que estes compram e têm renda, e representariam diferentes estratos da sociedade - assim, o cadeado continuaria fechado e com segurança.

Ainda com base no levantamento de Alba, Kraemer e Slongo (2012) verificou-se que a realização de experimentos está concentrada em alguns poucos programas de pós-graduação no Brasil. Parece ainda não existir uma tradição na formação de pesquisadores na área de marketing em experimentos (ou quase-experimentos). Isso pode levar a uma consequência, se considerarmos que doutores formados nos anos 90, assumindo que estes são os avaliadores mais constantes de artigos submetidos para congressos e periódicos no país, não 
receberam treinamento na condução de experimentos. Assim, talvez não tenham o conhecimento necessário para avaliar com rigor artigos que usam este método. Desse modo, a manipulação de dados em experimentos, que podem comprometer os resultados da pesquisa, por vezes pode ser negligenciada pelos avaliadores por desconhecimento dos critérios de qualidade desse processo, o que também seria aplicado à escolha da amostra dos grupos do experimento. A segurança oferecida pelo cadeado da pesquisa quantitativa neste caso estaria comprometida.

Jones e Suh (2000, p. 151) justificam o uso de estudantes no processo de coleta de dados do seu estudo da seguinte forma: "Não é incomum o uso de estudantes em estudos de pesquisa do consumidor e é apropriado quanto se testa esquemas teóricos onde o objetivo não seja generalizar para a população geral". Ou seja, eles apresentam os limites do uso deste tipo de amostra. Kau e Loh (2006) indicam o uso da técnica bola de neve para se obter respostas da população de não estudantes, quando da realização de uma coleta de dados online, junto a um banco de dados de alunos. Saídas e justificativas são possíveis, mas sempre que possível, para uma maior fidedignidade da análise de um fenômeno, escolher amostras que sejam representativas destes com consumidores reais.

Outro ponto que merece destaque no processo de amostragem é o uso de técnicas estatísticas paramétricas versus não paramétricas dependendo do tipo de amostra (probabilística - não probabilística). $\mathrm{O}$ pesquisador também deve observar os limites da pesquisa quantitativa neste ponto, e ter cuidado nas escolhas para os procedimentos de análises estatísticas. Apresentar uma justificativa ou mencionar a opção do uso de testes paramétricos em amostras não probabilísticas, embora já não seja novidade, deve ser demonstrado o conhecimento sobre os limites desta opção.

\section{A Questão das Medidas}

Jacoby (1978; p. 90) questionou: "qual implicação de um achado que se apresenta significante ou que a técnica estatística mais avançada tenha sido aplicada, se o instrumento de coleta de dados gerou dados inválidos?". Isto chama atenção para o cuidado com as métricas de marketing. Surpreendo-me, por vezes, ao revisar artigos submetidos a publicações acadêmicas nacionais, que ainda hoje há autores que elaboram instrumentos de mensuração sem o mínimo cuidado ou sistematização necessária. Às vezes é melhor 'tropicalizar' um instrumento já testado para mensurar determinando construto em outra língua do que desenvolver uma medida fraca, sem embasamento claro.

Churchill (1979; p. 64) comentou que aparentemente os especialistas em pesquisa de marketing estão se engasgando com suas medidas, no sentido de existência de uma lacuna entre medidas empregadas e a necessidade de medidas mais confiáveis. Tecnicamente falando, uma medida é válida "quando a diferença observada nos escores reflete diferenças verdadeiras nas características do que se está medindo e nada mais" (p. 65).

Tendo por base o artigo clássico de Churchill (1979), este autor recomenda que no desenvolvimento de medidas para a pesquisa na área de marketing, o investigador deve seguir as etapas de inicialmente especificar o domínio do construto (com base na revisão da literatura), gerar amostra de ítens relacionados ao construto (revisão da literatura, pesquisa de experiência, grupos de discussão, incidentes críticos), coletar dados e purificar as medidas (coeficiente Alfa e análise fatorial), coletar dados novamente, avaliar a confiabilidade (coeficiente alfa e confiabilidade split-half), verificar a validade (critério e matriz de métodos múltiplos) e, finalmente, estabelecer nor- 
mas (médias e outras medidas estatísticas que resumam a distribuição dos escores).

Um bom exemplo de desenvolvimento de medidas são os textos de Parasuraman, Zeithaml e Berry (1985, 1988). O primeiro enfatiza a relevância da teoria e o segundo indica os procedimentos para o desenvolvimento de uma escala bastante utilizada em pesquisas na área de qualidade em serviços. Aqui, os interessados no processo de construção de escalas, nos cuidados na sistemática e na validação de uma medida, encontram um texto ainda atual e bem escrito para se guiarem.

\section{Reflexões Finais}

Hall e Rist (1999), em um texto sobre métodos de pesquisa qualitativa, indicam que a pesquisa na área de marketing é incrementada quando múltiplos métodos são combinados na abordagem da triangulação. Desse modo, uma perspectiva para a qualidade na pesquisa quantitativa seria a triangulação de amostras, de técnicas estatísticas, de pesquisadores, etc. Certamente a flexibilidade nos processos aqui não se configura da mesma forma que no caminho qualitativo, mas certamente é possível incrementar os critérios de qualidade.

Critérios de qualidade que destacaria: revisão teórica que englobe o estado da arte do conhecimento na área, escolha de medidas consolidadas para o construto em avaliação, processo de amostragem adequado à problemática da pesquisa, atenção especial no processo de coleta de dados (e criação do banco de dados), escolha de técnicas estatísticas que atendam aos pressupostos do tipo de amostra e relações investigadas e, finalmente, mas não menos importante, honestidade para não manipular os resultados ou as interpretações destes. Outro aspecto por vezes esquecido e que traz qualidade a um estudo quantitativo consiste na articulação das análises com a teoria revisada. Caso exista a necessidade ou opção por desenvolvimento de medidas, seguir no mínimo o recomendado por Churchill (1979).

Certamente os manuais de pesquisa apresentam uma relação mais cuidadosa e aprofundada dos critérios de qualidade na pesquisa quantitativa. Aqui, apenas chamo a atenção para alguns básicos, que não são por vezes considerados. Há sete anos comentei em texto apresentado no Encontro de Marketing (EMA 2006) que o positivismo, tão atrelado aos métodos quantitativos, anteriormente predominante e justificável em determinado período da evolução do marketing, cede espaço para abordagens humanistas (FARIAS, 2006). Isso vai ao encontro do que defendi no artigo, de que a pesquisa em marketing ganha quando há flexibilidade nos métodos, no sentido de não excluir um caminho em detrimento de outro, por questões de puro desconhecimento. Acredito e defendo ser relevante expor, em especial aos alunos de doutorado na área de marketing, a métodos quantitativos e qualitativos, para que conheçam ambos, façam suas opções, mas não centrada na ignorância e sim em questões de afinidade e crenças.

A metáfora do cadeado indica que da mesma forma que este equipamento de segurança protege os indivíduos de invasões indesejáveis ou que suas posses sejam furtadas, a pesquisa quantitativa tem um mecanismo (procedimento) que leva, quando corretamente aplicado, a resultados numéricos que trazem segurança ao pesquisador e que são facilmente compreendidas com os números apresentados. Mas da mesma forma que um cadeado não é indestrutível, a pesquisa quantitativa pode ser contestada, em especial nos casos onde o indivíduo faz uso de técnicas de análises estatísticas sofisticadas, atendendo aos preceitos destas sem, no entanto, fazer uso do lado humano do pesquisador, e esquecendo que quem avalia trabalhos para publicações são também indivíduos que 
podem ter a chave para abrir o cadeado da segurança da pesquisa quantitativa. Tudo tem limite e o bom senso deve preponderar.

\section{Referências}

ALBA, G.; KRAEMER, F.; SLONGO, L. A. Panorama da Pesquisa Experimental em Marketing no Brasil. Anais do V EMA CD-ROM, Curitiba, PR., 2012.

CHURCHILL, G. A. Jr. A Paradigm for Developing Better Measures of Marketing Constructs. Journal of Marketing Research, v.16, n.1, p.64-73, 1979.

CHURCHILL, G. A. Jr. Marketing research: Methodological foundations, $7^{\text {th }}$ ed. The Dryden Press, New York, 1999.

COSTA, A. L. C. N. da; FARIAS, S. A. de. $\mathrm{O}$ aroma ambiental e sua relação com as avaliações e intenções do consumidor no varejo. Revista de Administração de Empresas online, v.51, n.6, p. 528-541, 2011.

FARIAS, S. A. de. Pesquisa Flexível em Marketing: o lado humanista de uma ciência socialmente construída e fixamente investigada. Anais do EMA 2006 CDROM - Encontro de Marketing da ANPAD. Rio de Janeiro, RJ, 2006.

HALL, A. L.; RIST, R. C. Integrating Multiple Qualitative Research Methods (or avoiding the precariousness of a onelegged stool). Psychology \& Marketing, v.16, n.4, p. 291-30, 1999.

JACOBY, J. Consumer research: How valid and useful are all our consumer behavior research findings? Journal of Marketing, v.2, p. 87-95, 1978.

JONES, M. A.; SUH, J. Transactionspecific satisfaction and overall satisfaction: an empirical analysis. Journal of Service Marketing, v.14, n.2, p.147159, 2000.

KAU, A.-K.; LOH, E. W.-Y. The effects of service recovery on consumer satisfaction: a comparasion between compainants and non-complainants. Journal of Service Marketing. v.20, n.2, p. 101-111, 2006.

MAZZON, J. A.; HERNANDEZ, J. M. C. Produção Científica Brasileira em Marketing no Período 2000-2009. Revista de Administração de Empresas, v.53, n.1, p. 67-80, 2013.

PARASURAMAN, A.; ZEITHAML, V. A.; BERRY, L. A conceptual model of service quality and its implications for future research. Journal of Marketing, v.49, p. 41-50, 1985.

PARASURAMAN, A. ZEITHAML, V. A.; BERRY, L. SERVQUAL: a multiple item scale for measuring consumer perceptions of service quality. Journal of Retailing, v.64, n.1, p.12-40, 1988.

RICHARDSON, L. Writing a method of inquiry. In Denzin, N. K., and Lincoln, Y. S. (Eds), Handbook of Qualitative Research, Sage, Newbury Park, CA, chap 32, p. 516-529, 1994.

VIEIRA, F. G. D. Por quem os sinos dobram? Uma análise da publicação científica na área de marketing do ENANPAD. Anais do ENANPAD, Foz do Iguaçu, PR, 1998. 